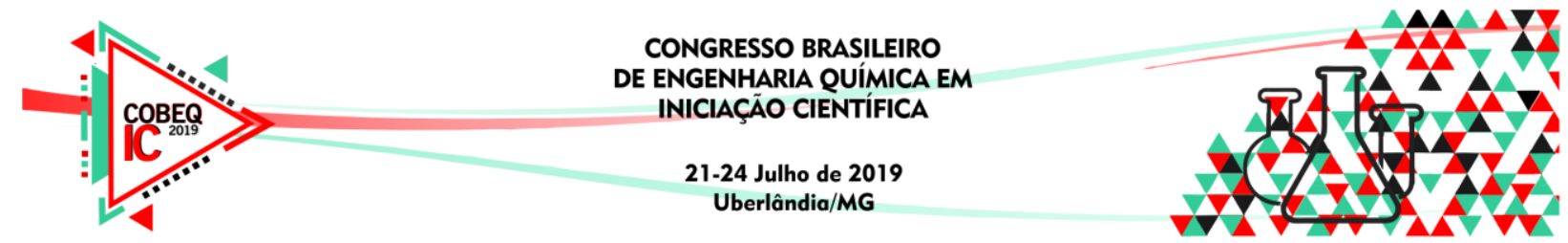

\title{
ESTUDO DA INFLUÊNCIA DA CONFORMAÇÃO E TEMPERATURA NA AMILOSE POR MODELAGEM MOLECULAR
}

\author{
E. MOCHIUTTI ${ }^{1}$, J. P. O. LIMA ${ }^{1}$, A. L. S. CARVALHO ${ }^{1}$, A. E. S. NASCIMENTO ${ }^{1}$, M. C. \\ MARTELLI $^{1}$ \\ ${ }^{1}$ Universidade Federal do Pará, Faculdade de Engenharia Química \\ E-mail para contato: mochiuttieric@gmail.com
}

\begin{abstract}
RESUMO - Simulações computacionais são a alternativa menos custosa para realizar análises em ramos da ciência dos materiais. Nesse trabalho, o objetivo foi simular a estrutura de amilose solvatada em água para analisar parâmetros com base na mudança de temperatura e conformação no sistema. A dinâmica molecular foi feita no software GROMACS com uma estrutura construída anteriormente no software Glycam carbohydrate builder e otimizada no Spartan Wavefunction V.8. Como resultados, percebeu-se que a temperatura possui influência direta na energia cinética do sistema, enquanto a torção alterou a energia potencial e consequentemente a energia total do sistema.
\end{abstract}

\section{INTRODUÇÃO}

O biofilme é um material produzido a partir de um componente biológico que tem como função, ser uma barreira contra o ambiente externo. (LUCHESE et al., 2015). Amido é um polímero biodegradável com potencial termoplástico quando na presença água ou um plastificante (geralmente poliol ou glicerol). O material resultante pode ser produzido com as mesmas tecnologias já utilizadas em plásticos sintéticos. (GARCÍA et al., 2011).

Amilose e amilopectina são as estruturas majoritárias presentes em todos os tipos de amido encontrados na natureza. A amilose, que representa cerca de 15 a $35 \%$ dos grânulos na maioria das plantas, é um polissacarídeo em maior parte linear com D-glucoses ligadas por ligação $\alpha$-(1-4). Ainda sobre a estrutura, amiloses tendem a formar uma conformação de dupla hélice (amilose ligado a amilose) ou de uma hélice (quando em presença de complexante) (PÉREZ et al., 2010).

Em estudos de ciência dos materiais, várias das análises experimentais precisam ser realizadas para caracterizar, medir e manipular as propriedades que a estrutura possa apresentar. Desta forma, alguns experimentos tornam-se inviáveis em algumas situações, como quando se faz necessário expor o material a temperaturas e pressões extremamente altas ou a um reator nuclear. Sendo, simulações computacionais podem ser uma alternativa mais simples para obter os mesmos resultados (HILL et al., 2005)

O trabalho teve como objetivo realizar uma simulação computacional, analisando a conformação da molécula de amilose baseada em suas torções e realizar a dinâmica molecular 


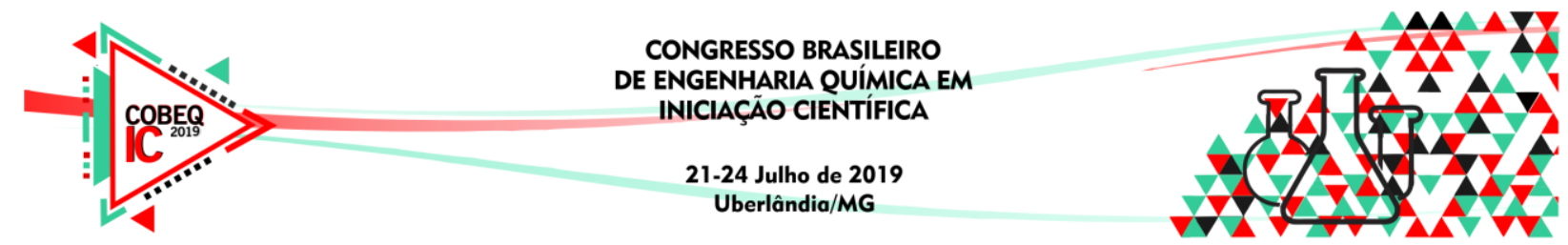

do sistema formado por amilose e água, obtendo assim, resultados de interação entre as estruturas e observar como o polímero reage a solvatação e à mudança de temperatura no sistema.

\section{MATERIAIS E MÉTODOS}

As moléculas de amilose foram construídas com 12 anéis de $\alpha$-D-glucose em uma cadeia linear $\left(\mathrm{C}_{72} \mathrm{H}_{122} \mathrm{O}_{61}\right)$, assim dispondo de 11 ligações $\alpha-(1-4)$, com o auxílio do software Glycam carbohydrate builder, variando a torção das ligações entre os monômeros entre $60^{\circ}$ a $180^{\circ}$, variando em $5^{\circ}$. A Figura 1 representa a estrutura em 2D da Amilose.

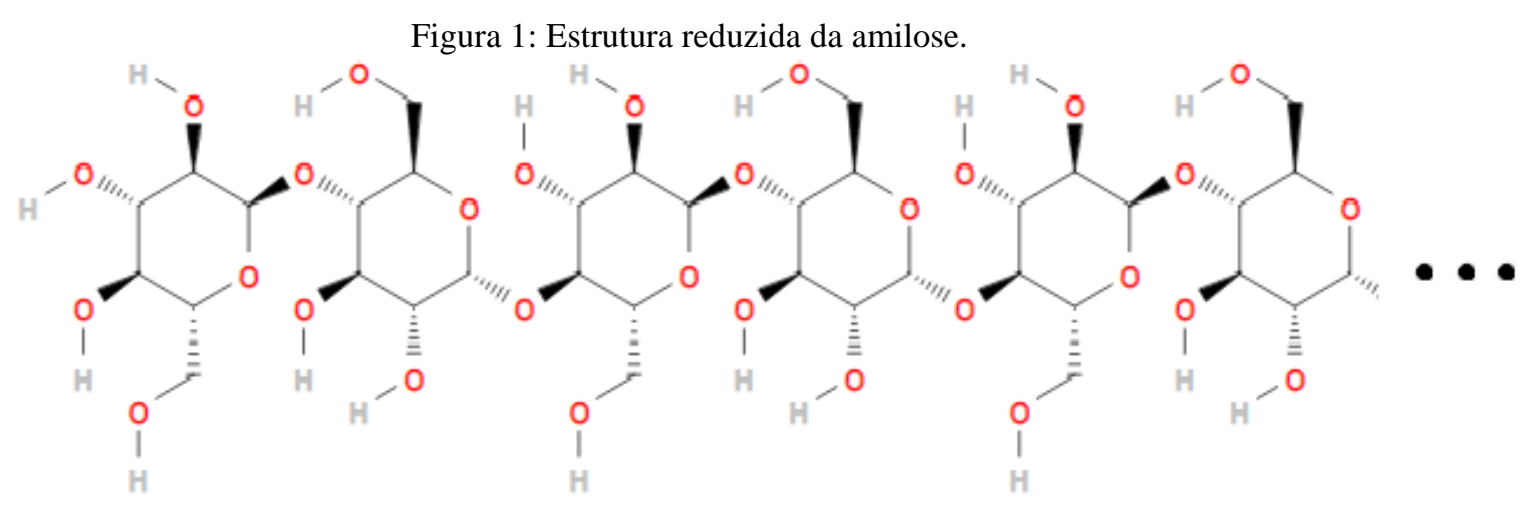

Utilizando o software Spartan Wavefunction V.8 realizou-se o cálculo de minimização de energia estrutural de cada conformação, através da aplicação de um método semi-empírico com base PM6 (HOSTAŠ et al., 2013). Em seguida, foram selecionadas, para as próximas etapas de dinâmica, a conformação de menor energia e a conformação linear. A dinâmica molecular no software GROMACS com o campo de força reotimizado para carboidratos GROMOS 56ACARBo adaptado por HANSEN e HÜNENBERGER (2010).

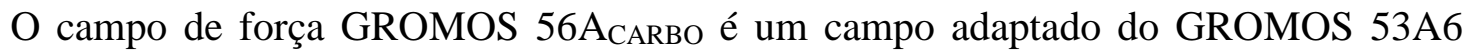
para carboidratos baseados em hexopiranose restrito para estruturas contendo apenas átomos de carbono, oxigênio e hidrogênio, contendo apenas ligações simples, sem funções oxigenadas além de álcool, éter, hemiacetal, acetal e sem componentes cíclicos que não sejam anéis de 6 átomos. (HANSEN E HÜNENBERGER, 2010).

O sistema foi montado como uma caixa cúbica de dimensões $60 \times 60 \times 60$, sendo solvatado com 6948 moléculas de água de modelo SPC (Simple Point Charge) (PULLMAN, 1981), contendo condições periódicas de contorno nos eixos XYZ, o qual demonstra multiplas repetição da caixa cúbica de solvatação nas 3 dimensões.

Antes do cálculo de dinâmica, um cálculo de minimização foi feito a partir de um algoritmo steepest descent com um total de 50000 passos. Posteriormente, foram realizadas duas etapas de equilíbrio, NVT, que consiste em um sistema formado a partir de número de mols, volume e temperatura constantes, e NPT, com número de mols, pressão e temperatura constantes. Por fim, a dinâmica molecular foi executada seguindo parâmetros previamente estabelecidos. Essa simulação foi efetuada com $10^{7}$ passos para um tempo de $20 \mathrm{~ns}$ (nanossegundo), baseando-se no algoritmo de integração Leap-Frog em condições periódicas 


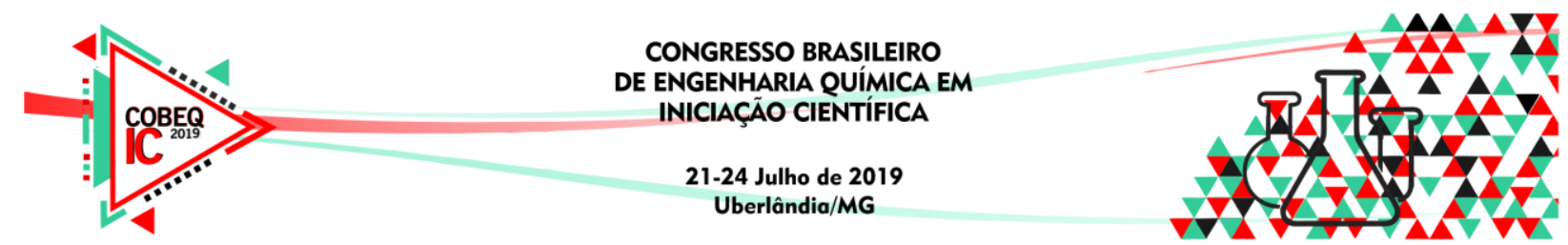

de contorno em 3 dimensões. Foi especificado também o método de cálculo de interações a longa distância, sendo esse o PME (Particle Mesh Ewald) com uma restrição de 1,4 nm de alcance eletrostático e de Van der Waals. O controle de pressão foi efeito a partir de um barostato de Parrinello-Rahman com temperatura de referência de 1 bar. Todas essas dinâmicas foram executadas com temperatura 298,15 K; 323,15 K e 348,15 K estabelecidas com um termostato de V-rescale.

\section{RESULTADOS E DISCUSSÃO}

A Figura 2 mostra a representação tridimensional das estruturas de $180^{\circ}, 160^{\circ}, 140^{\circ}, 120^{\circ}, 100^{\circ}$ e $80^{\circ}$ (da esquerda para direita) e Figura 3 contém o gráfico de energia por ângulo da torção da cadeia.

Figura 2: Conformações das Amiloses de (a) $=180^{\circ},(\mathrm{b})=160^{\circ},(\mathrm{c})=140^{\circ},(\mathrm{d})=120^{\circ},(\mathrm{e})=100^{\circ} \mathrm{e}$ (f) $=80^{\circ}$, da esquerda para direita

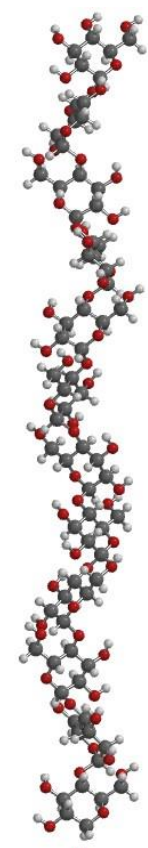

(a)

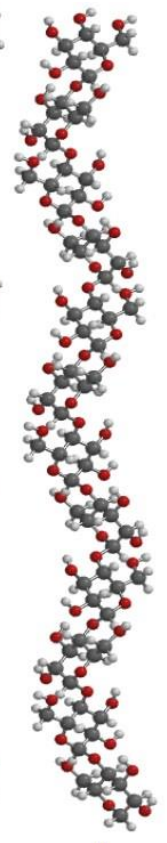

(b)

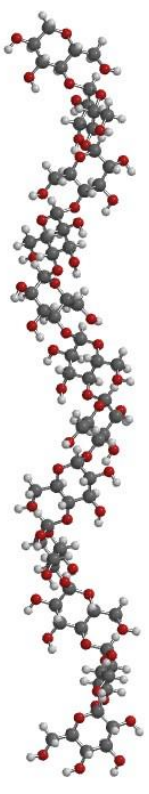

(c)

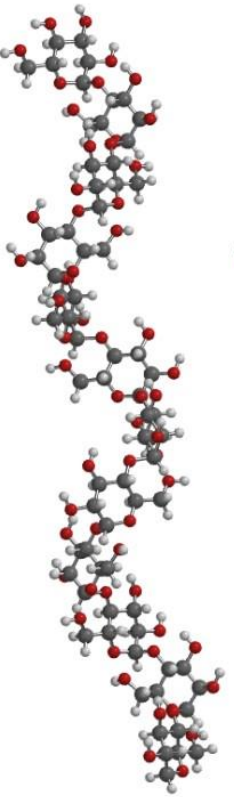

(d)

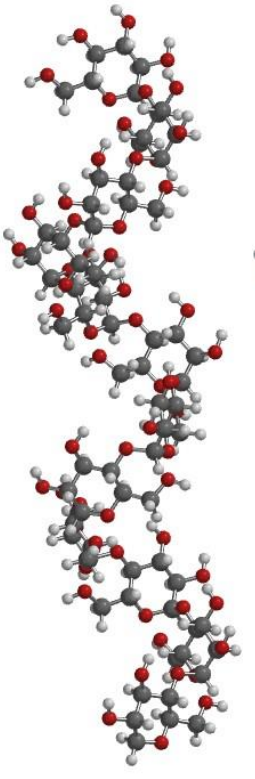

(e)

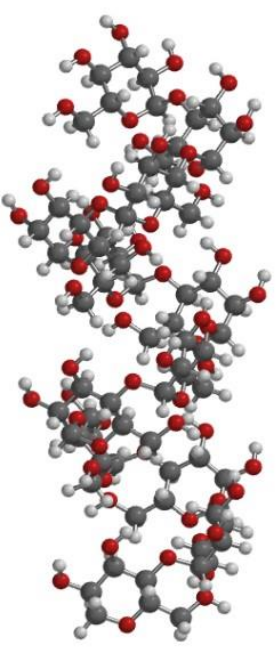

(f)

Figura 3: Análise da energia das Conformações Amiloses $180^{\circ}$ a $70^{\circ}$.

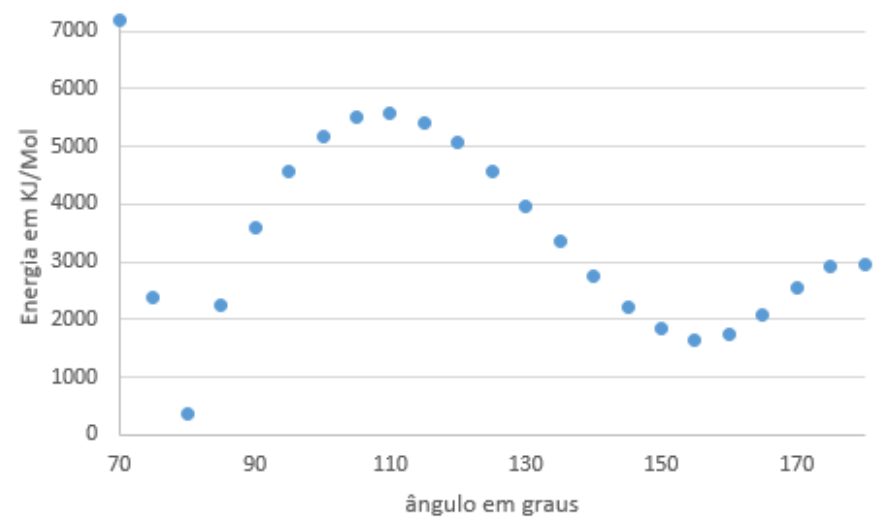




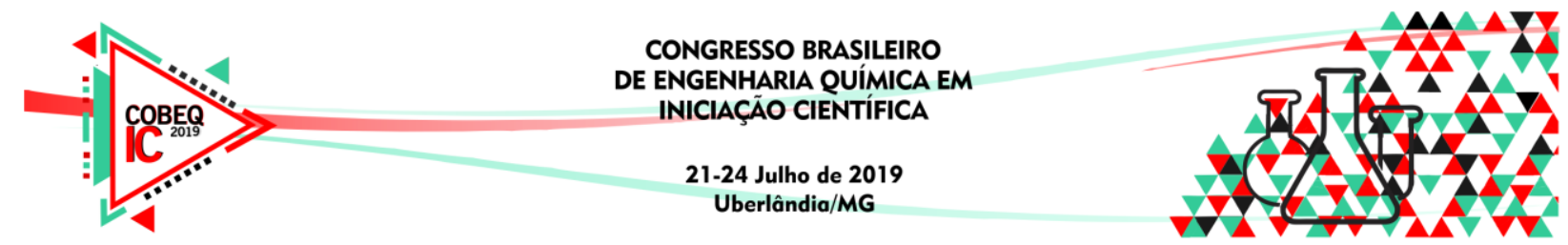

A Figura 3 mostra o ângulo que as conformações foram geradas e a energia de cada uma. Os ângulos abaixo de $60^{\circ}$ e $65^{\circ}$ obtiveram resultados de energia nos valores de $3443004,7 \mathrm{~kJ} / \mathrm{mol}$ e $11718243,96 \mathrm{~kJ} / \mathrm{mol}$ respectivamente, mostrando que as energias nessas conformações são altíssimas. Para a angulação de $80^{\circ}$ obteve-se $361,95 \mathrm{~kJ} / \mathrm{mol}$, valor que apresentou a menor energia de conformação estrutural. Para as etapas de dinâmica molecular selecionou-se as conformações de $180^{\circ}$ e $80^{\circ}$. Na Figura 4 estão as figuras das caixas de simulação para dinâmica molecular.

Figura 4: Caixa de simulação para os sistemas solvatados com as conformações de $180^{\circ}$ e $80^{\circ}$.

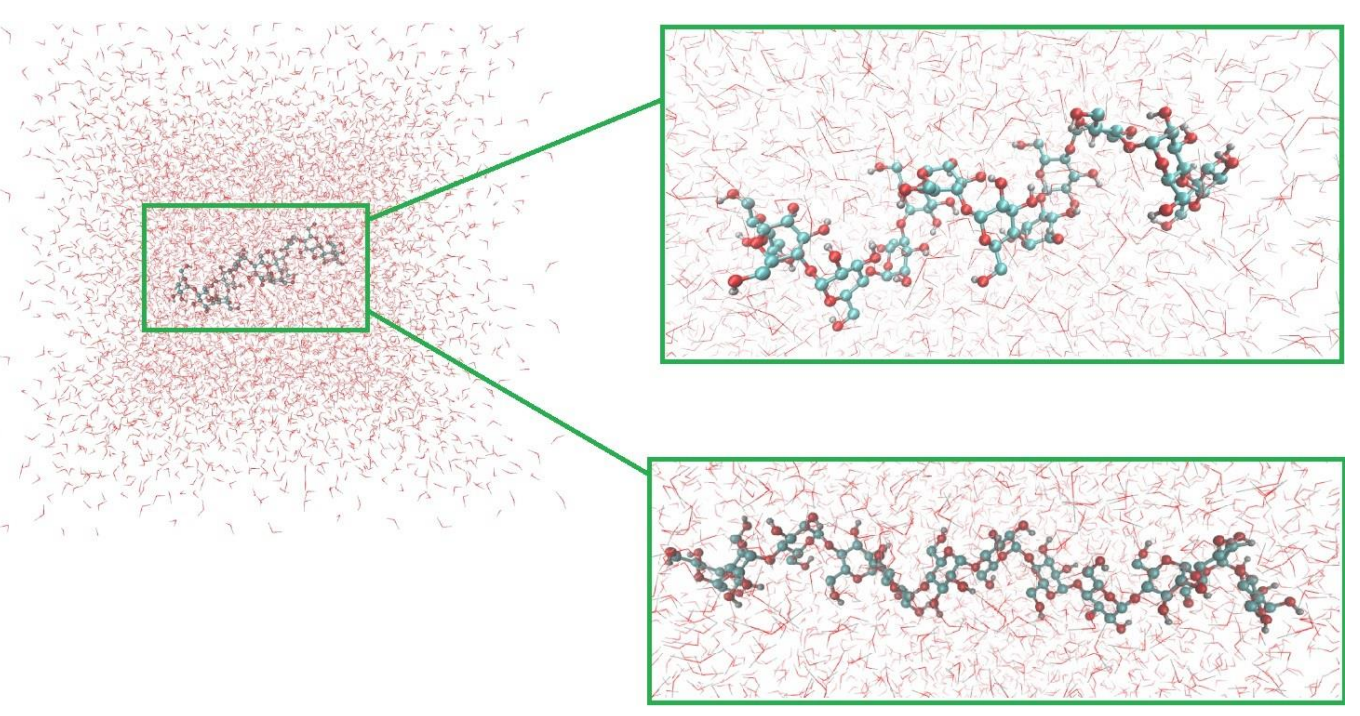

A Figura 5 (a) mostra a energia cinética no decorrer da simulação, e a Figura 5 (b) demonstra a energia potencial dos sistemas montados ao longo da simulação.

Figura 5: (a) Energia Cinética e (b) Energia Potencial dos sistemas.
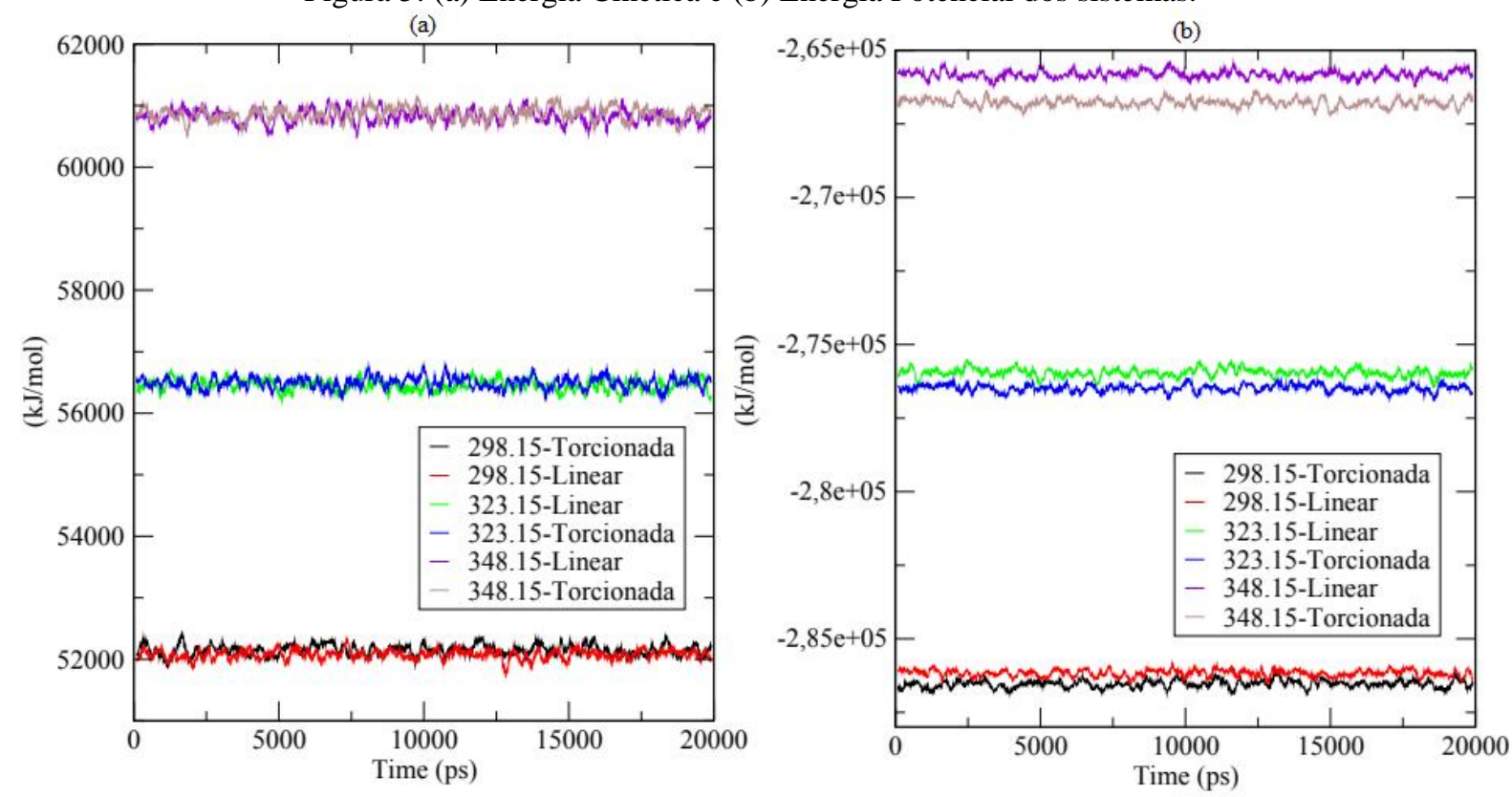


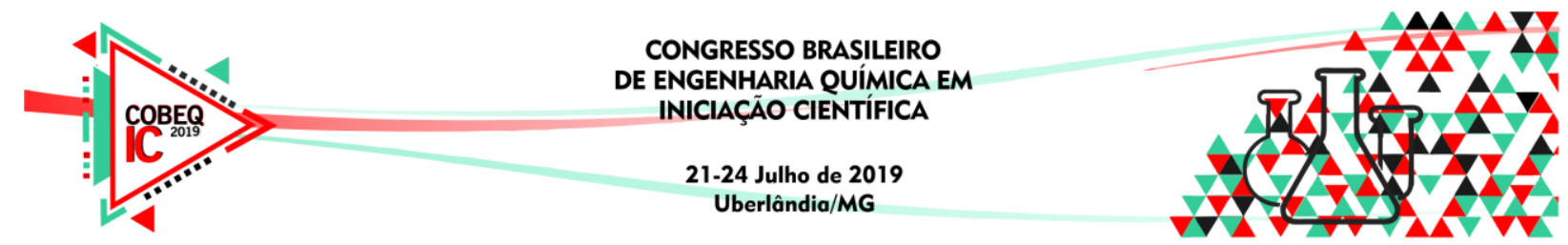

A Figura 5 (a) mostra que existe um aumento da energia cinética com o aumento da temperatura do sistema, independentemente da conformação inicial. Porém, os resultados da energia potencial, Figura 5 (b), mostram que há diferença perceptível entre os de sistemas com amilose torcionada $\left(80^{\circ}\right)$ e linear $\left(180^{\circ}\right)$, onde a amilose torcionada apresentou menor energia potencial.

A Figura 6 apresenta os gráficos de energia total dos sistemas ao longo da dinâmica de 20 nano segundos.

Figura 6: Energia total dos sistemas (em $10^{5} \mathrm{~kJ} / \mathrm{mol}$ ) nas três temperaturas com estrutura linear e torcionada.

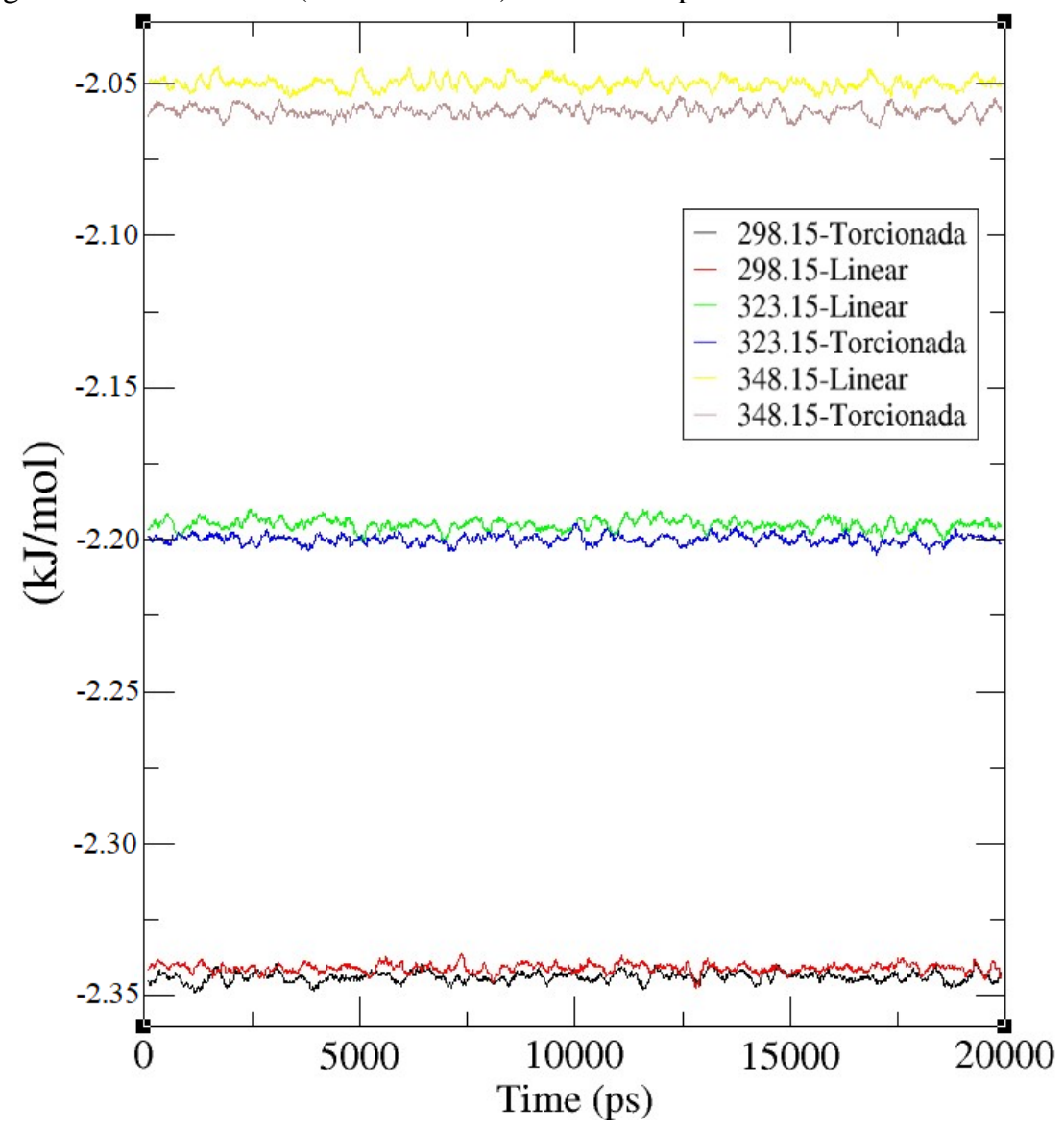

Analisando a Figura 6, é possível observar a conservação da energia total do sistema que segundo Picinin (2007), a conservação de energia total é um parâmetro importante para verificar se a simulação foi realizada corretamente. É possível observar também que os valores das simulações realizadas com a estrutura com torção de $180^{\circ}$ apresentou energia total maior comparada com a equivalente com torção de $80^{\circ}$.

\section{CONCLUSÃO}

A partir das análises de energia entre diferentes torções $\left(60^{\circ}\right.$ a $\left.180^{\circ}\right)$, obteve-se que a menor energia foi obtida por uma conformação de $80^{\circ}$ (torcionada). Assim, quando comparado com a de $180^{\circ}$ no cálculo de energia por dinâmica molecular, observa-se que a torcionada tende a ter uma menor energia. Os sistemas mostraram que para resultados de 


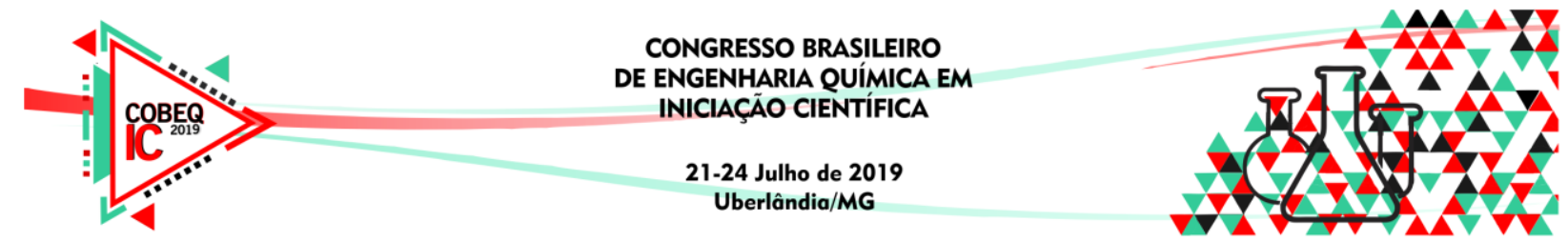

energia potencial e energia total há diferença significativa entre valores obtidos de amiloses torcionadas e linear. Além disso, a temperatura teve influência direta na energia cinética, de forma que à medida que a temperatura aumenta, a energia cinética aumenta, não havendo mudança relacionada a conformação. Portanto pode-se concluir que a conformação inicial da simulação altera a energia potencial do sistema estudado.

\section{REFERÊNCIAS}

GARCÍA, N. L., RIBBA, L., DUFRESNE, A., ARANGUREN, M., GOYANES, S. Effect of glycerol on the morphology of nanocomposites made from thermoplastic starch and starch nanocrystals. Carbohydrate Polymers, v. 84, p. 203-210, 2011.

HANSEN, H. S., HÜNENBERGER, P. H. A reoptimized GROMOS force field for hexopyranose-based carbohydrates accounting for the relative free energies of ring conformers, anomers, epimers, hydroxymethyl rotamers, and glycosidic linkage conformers. Journal of Computational Chemistry, v. 32, p. 998-1032, 2010.

HILL, J. R., SUBRAMANIAN, L., MAITI, A. Molecular modeling techniques in material sciences. Boca Raton: Taylor \& Francis, 2005.

HOSTAS̆, J.; ŘEZÁČ́, J.; HOBZA, P. On the performance of the semiempirical quantum mechanical PM6 and PM7 methods for noncovalent interactions. Chemical Physics Letters, v. 568-569, p. 161-166, 2013.

LUCHESE, C. L., FRICK, J. M., PATZER, V. L., SPADA, J. C., TESSARO, I. C. Synthesis and characterization of biofilms using native and modified pinhão starch. Food Hydrocolloids, v. 45, p. 203-210, 2015.

PÉREZ, S., BERTOFT, E. The molecular structures of starch components and their contribution to the architecture of starch granules: A comprehensive review. Starch Stärke, v. 62, p. 389-420, 2010.

PICININ, A. Simulações por dinâmica molecular aplicadas ao estudo de defeitos em cristais coloidais bidimensionais. Tese (doutorado em Física) -Instituto de física de São Carlos. Universidade de São Paulo, São Carlos. 2007.

PULLMAN, B. (Ed.). Intermolecular Forces. Jerusalém: The Jerusalem Symposia on Quantum Chemistry and Biochemistry, 1981. 